\title{
Une pratique nourrie par l'expérience personnelle
}

\section{Anne-Françoise Allaz}

Prof. Dr méd., membre de la rédaction

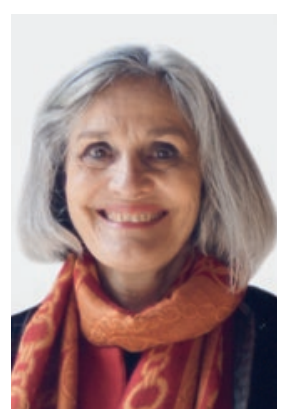

En pratique clinique, la rencontre avec un patient se tisse bien souvent entre savoir et incertitude, objectivité et subjectivité. Parallèlement aux aspects bio-médicaux fondés sur la science et l'évidence, il n'est plus à démontrer que les dimensions psychosociales, contextuelles et culturelles sont des déterminants centraux de la santé. Les sciences humaines ont par conséquent un rôle essentiel à jouer pour contrebalancer la place occupée en Occident par la technologie et la standardisation dans la pratique et la formation des professions médicales. Elles y sont d'ailleurs de mieux en mieux intégrées aujourd'hui. Elles donnent des clefs pour répondre aux questionnements et aux attentes de nos patients et contribuent à faciliter la résolution des problèmes complexes auxquels nous sommes confrontés. Un exemple d'actualité: le conseil vaccinal à une personne non convaincue qui, au-delà des évidences bio-épidémiologiques, comprend des facettes pédagogiques, de communication, de psychologie, d'éthique, entre autres.

L'approche narrative, développée notamment à la suite des travaux de Rita Charon, médecin et directrice du programme de médecine narrative de l'Université de Columbia, utilise l'écriture réflexive comme outil pour se rapprocher de l'expérience vécue des patients face à la maladie [1]. Ceux-ci sont invités à raconter ou écrire leur histoire, leurs émotions, leurs préoccupations. Le bénéfice subjectif de cette approche dans le contexte de maladies très diverses est aujourd'hui avéré et a fait l'objet de revues dans des journaux de renom [2]. Elle renforce également la reconnaissance de la singularité des patients et la précieuse alliance thérapeutique. Inspirée par ce modèle, notre équipe d'un service de médecine de réhabilitation des Hôpitaux universitaires de Genève a demandé à des patients âgés fortement atteints dans leurs capacités fonctionnelles d'écrire comment ils envisageaient leur retour à domicile. Loin de l'inquiétude teintée de pessimisme de leurs soignants, les personnes âgées sollicitées - bien que très atteintes dans leur santé - nous ont confié un surprenant florilège de projets optimistes et joyeux, tels que des déplacements ou voyages pour voir un proche, la reprise d'activités sociales appréciées, ou la simple réintégration d'un domicile ressenti comme un havre de bien-être et de tranquillité. Cette expression de désir et de rêves nous a permis de mieux comprendre leur vision d'euxmêmes. En parler a contribué à renforcer l'engagement mutuel dans les soins. Ces récits nous ont également beaucoup touchés. C'est effectivement un bénéfice des approches narratives d'induire de l'empathie, en connectant les soignants à la dimension personnelle et humaine des patients qui les consultent [3]. Or, nous savons combien l'empathie est un ingrédient essentiel de la rencontre. "On ne voit bien qu'avec le cour. L'essentiel est invisible pour les yeux», disait le Petit Prince de Saint-Exupéry.

Les approches réflexives soulignent que l'expérience se construit aussi en exerçant un recul critique sur ses propres actions et émotions.

Parmi les moyens de renforcer l'épaisseur d'humanité de la relation de soin, les approches réflexives offrent des perspectives très enrichissantes. Elles soulignent que l'expérience se construit à partir de la pratique, mais aussi en exerçant un recul critique sur ses propres actions et émotions. Il est par exemple frappant de voir combien les travaux d'écriture réflexive proposés aux étudiants en médecine leur permettent d'élargir leurs points de vue sur soi et sur l'autre. En pratique clinique, lorsque la relation médecin-malade est difficile, prendre conscience de son vécu personnel et l'utiliser pour enrichir la rencontre ou encore oser "ne pas savoir» en remplaçant une position de maîtrise par celle d'un questionnement partagé sont des démarches particulièrement fécondes [4].

$\mathrm{Au}$ vu de leurs bénéfices sur le développement professionnel et sur la relation avec les patients, les compétences narratives et réflexives que Rita Charon nomme "une pratique nourrie par l'expérience personnelle» mériteraient de faire plus systématiquement partie des curriculae de formation.

\section{Références}

1 Charon R, Hermann N. A Sense of Story, or Why Teach Reflective Writing? Acad Med. 2012;87:5-7.

2 Fioretti C, Mazzocco K, Riva S, et al. Research studies on patients' illness experience using the Narrative Medicine approach: a systematic review. BMJ Open. 2016;6:e011220. doi:10.1136.

3 Baarts C, Tulinius C, Reventlow S. Reflexivity - A strategy for a patient-centred approach in general practice. Family Practice 2000;17:430434.

4 Widmer D, Allaz AF. Outils pour la réflexivité en psychosomatique: Qu'est-ce qui a changé en 30 ans? Rev Med Suisse. 2017;13:189. 